\title{
Image Resolution Enhancement Method Based on Feature Space
}

\author{
Liqun Wang, ${ }^{1}{ }^{*}$ and Honghui Fan ${ }^{2}$ \\ ${ }^{1}$ School of Information Engineering, Northeast Dianli University, Jilin, Jilin, 132012, China \\ ${ }^{2}$ Computer Engineering School, Jiangsu University of Technology, Changzhou Jiangsu, 213001, \\ China \\ aguoshuqiang@gamil.com
}

Keywords: Resolution enhancement; Feature Space; Color image; Image process

\begin{abstract}
In this paper, an approach for resolution enhancement of color image based on feature space method is proposed. Many images, such as landscape, natural scene and so on, are fractal, and they can often be assumed as self-correlation. Focusing on this point, we describe the image by feature space. This method reflects the characteristics of color image, and realizes the image interpolation by using the feature space. Experiment result shows that this method can enhance the image resolution more effectively comparing with nearest neighbor interpolation, bilinear interpolation.
\end{abstract}

\section{Introduction}

Image resolution enhancement is a method of signal processing and image processing. It can transform an existing low resolution image into high resolution image by using the software algorithm. In video monitoring, image printing, forensic analysis, medical image processing, satellite imaging and other fields, image resolution enhancement has been applied widely. Image resolution enhancement related to some basic problem in many fields such as image processing, computer vision, optimization theory. Resolution enhancement has become one of the hot spots in the research field and it has important significance to image processing [1].

Image resolution enhancement is an image data regeneration process within a given range of space. The more image data can be estimated by the finite discrete image data accurately to construct observation images with higher resolution to reflect the real scene. Advanced resolution enhancement technique can effectively enhance the image resolution, so it is widely used in satellite remote sensing, material analysis, medical diagnostic, traffic management, criminal investigation and so on.

Resolution enhancement is divided into the traditional interpolation methods and modern interpolation method. The traditional interpolation method is also known as linear interpolation that uses the known image pixel gray value and special interpolation function to calculate the unknown pixel gray value. The typical linear interpolation methods are nearest neighbor interpolation, bilinear interpolation, bicubic interpolation, polynomial interpolation and spline interpolation algorithm [2-6]. The high frequency information of the image is inhibited by traditional interpolation method, so the blur or aliasing phenomenon would appear in the edge region of restoration images, and it is not ideal to restore the edge and texture features of the image. The modern interpolation method is also called nonlinear interpolation, and it introduces the fractal topology, wavelet analysis, partial differential equations, nonlinear optimization theory etc. into digital image processing [7-10]. The modern interpolation methods can use different interpolation method in different regions of image according to the regulation of the image spectrum weighted coefficient.

In this paper, the local images are cut out form the color image, such as landscape image, texture image, building image and so on. From these local images, a similar pattern can be extracted by a specific feature space, and the pattern can express the image compactly. Using this pattern we propose a method to enhance the image resolution. 


\section{Image Pattern Extracted by Feature Space}

The color images are fractal generally, so the image pattern can be extracted by feature space to express the images. As shown in Fig. 1, sample windows are cut out from image, and then the image vector is generated by scanning the sample window. The image vector can be given as:

$$
X=\left[\begin{array}{llll}
x_{1} & x_{2} \cdots & x_{R}
\end{array}\right], x_{i}=\left[\begin{array}{llll}
f(x)_{00} & f(x)_{01} \cdots & f(x)_{m n}
\end{array}\right]^{T}
$$

where $R$ is the number of sample windows, $\mathrm{m}$ and $\mathrm{n}$ are the width and height of sample window, $f(x)_{m n}$ is the gray value in position $(\mathrm{m}, \mathrm{n}), X$ is the image vector. Then the variance matrix of image vector $X$ can be obtained and using KL transform the Eigen vectors column can be given as:

$$
E=\left[\begin{array}{lll}
e_{1} & e_{2} \cdots & e_{D}
\end{array}\right], e_{i}=\left[\begin{array}{lll}
e_{1} & e_{2} \cdots & e_{m n}
\end{array}\right]_{i}^{T}
$$

where $D$ is the dimension of Feature space. The cumulative proportion is calculated as:

$$
\eta_{(D)}=\sum_{i=1}^{D} \lambda_{i} / \sum_{i=1}^{R} \lambda_{i}
$$

In feature space method, although the dimension is smaller than the rank of the image vector sequence, it can represent the learning samples, because it is often scattered in a particular direction rather than messy. It may also be considered that the learning samples are high correlation. That is, if cumulative proportion is obtained, the eigenvector can be considered as extracted image pattern, and the defective image can restored by extracted image pattern.

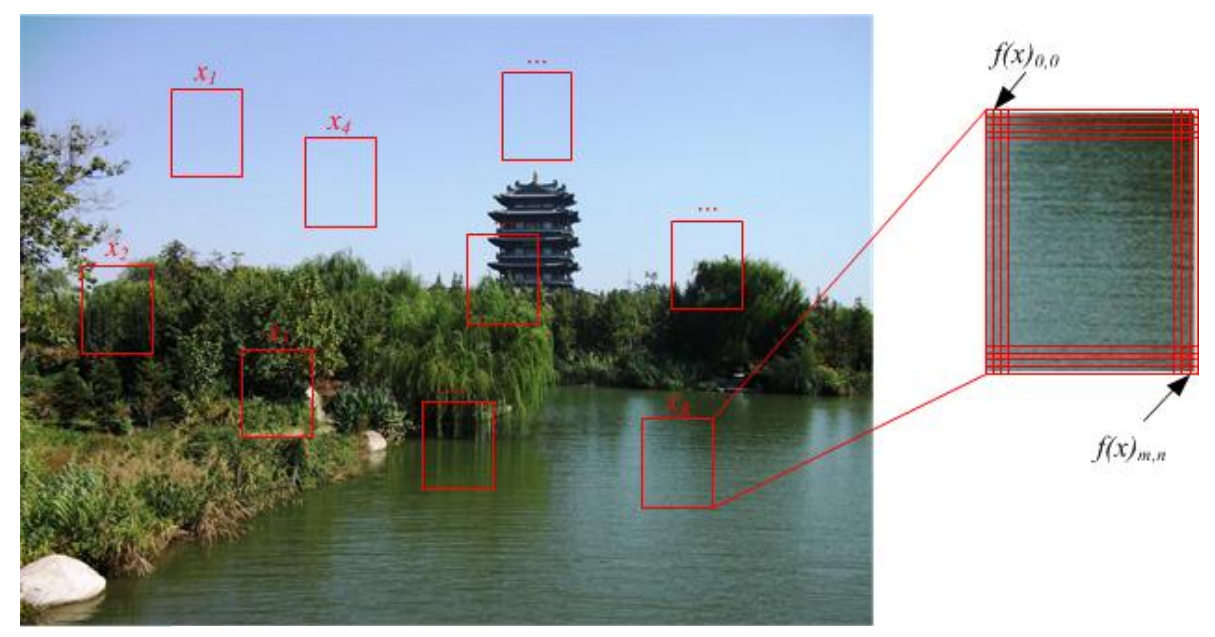

Figure 1. Sample windows cut out from image

\section{Resolution Enhancement Using Feature Space}

Image interpolation is performed by image pattern that expresses the image using eigenvectors. The image vector generated by sample windows is projected onto the Eigen space. The projection point is given as:

$$
p_{i}=E^{T} x_{i}
$$

If there is defect, open hole in sample image, the part of defect is expressed by 0 . So,

$$
\hat{x}_{i}=\left[\begin{array}{lll}
f(x)_{00} & f(x)_{01} \cdots & f(x)_{m n}
\end{array}\right]^{T}=\left[\begin{array}{ccc}
1 & \cdots & 0 \\
\vdots & \ddots & \vdots \\
0 & \cdots & 1
\end{array}\right] x_{i}=\Sigma x_{i}
$$

Whrer $\Sigma$ expressed whether is there the defect parts in the sample windows. When the image vector projects to feature space, the projection point can be transformed as:

$$
\hat{p}_{i}=E^{T} \hat{x}_{i}
$$

If the cumulative proportion is enough higher, the approximation is sufficient in local areas that have been learned deeply, and if the image has strong autocorrelation property, the approximation is also sufficient in other areas of the given image. So, the relationship of the projection point $\hat{p}$ of defect pixel and the projection point $p$ is given as: 


$$
\hat{p}_{i} \propto E^{T} \Sigma E p_{i}=T p_{i}
$$

In combination with the former equation, the defect pixels can be calculated by the following equation:

$$
\hat{x}_{i}=E T^{-1} E^{T} x_{i}
$$

\section{Experimental Results}

In order to validate the feasibility of the resolution enhancement and superiority in high frequency region, the experiment is carried out. The peak signal to noise ratio $(P S N R)$ is used to evaluate the resolution enhancement effectiveness based on feature space. The formula of PSNR is given as:

$$
P S N R=10 \lg \frac{255^{2} \times q \times m \times n}{\|\hat{x}-x\|^{2}}
$$

where $\hat{x}$ is the reconstruction image, and $x$ is the original image, $q$ is the enlargement factor.

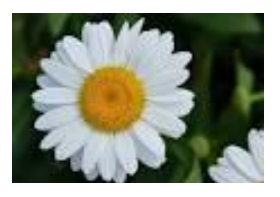

Figure 2. (a) The original image

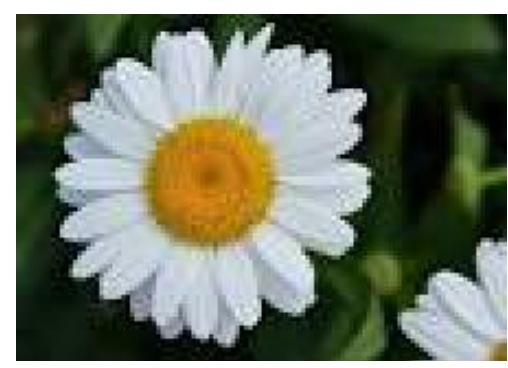

Figure 2. (b) Nearest neighborhood interpolation

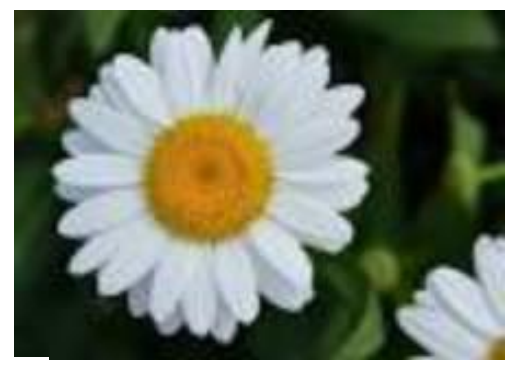

Figure 2. (c) Bilinear

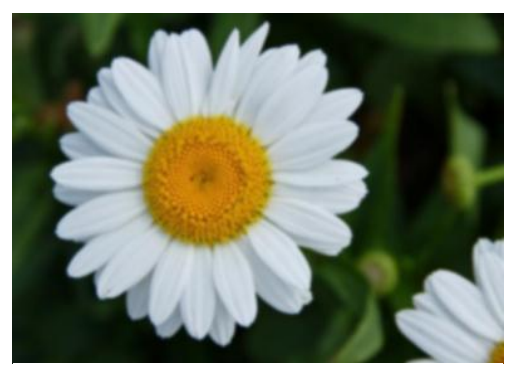

Figure 2. (d) Feature space method

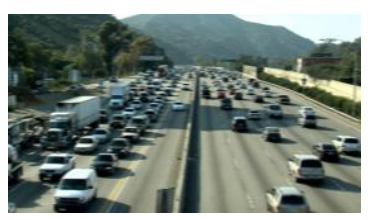

Figure 3.(a) Image captured from video

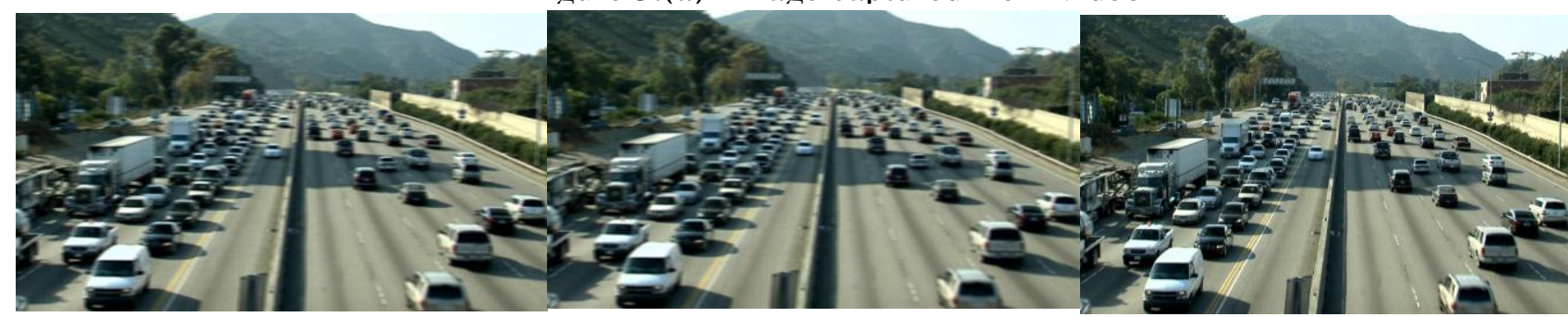

Figure 3.(b) Nearest neighborhood interpolation
Figure 3.(c) Bilinear
Figure 3.(d) Feature space method

In this paper, a flower image and a frame image which is cut out from a blurred, and lower quality of video are enhanced by following methods: (1) neighborhood interpolation; (2) bilinear interpolation; (3) feature space method. Fig. 3 (a) is the original image, and its size is $112 \times 80$ pixels. It is very representative because there are so many texture and edges. Fig. 3 (a) is a frame taken from 
a low resolution video in actual environment whose size is $200 \times 150$ pixels. The experimental results are shown in Fig. 2, Fig. 3 and Table 1.

Table 1 The PSNR of the three methods

\begin{tabular}{|c|c|c|c|}
\hline & Neighborhood interpolation & Bilinear interpolation & Feature space method \\
\hline Flower & 43.27 & 45.62 & 52.33 \\
\hline Video & 38.21 & 41.81 & 49.46 \\
\hline
\end{tabular}

In these two sets experiments, the results of nearest neighbor interpolation are unacceptable. Although its computational speed is very fast and the algorithm is simple, it only considers the pixel with maximum impact on it without considering the influence of other adjacent pixels. As shown in Fig. 2 (b), Fig. 3 (b) and Table 1, the PSNR of nearest neighbor interpolation is the worst because its discontinuity.

Bilinear interpolation considers contribution factor of the pixels around the center point, so it avoid the discontinuous disadvantages of nearest neighbor interpolation. However, the amount of calculation increases. Furthermore this method would generally retain the low-frequency signal components and lost the part of the high-frequency signal components. Therefore, it will make the edges of the interpolated image fuzzy. As shown in Fig. 2 (c) and Fig. 3 (c), the reconstruction image using this bilinear interpolation still has a small amount of "grid" effect.

As shown in Fig. 2, Fig. 3 and Table 1, the resolution enhancement using feature space can improve resolution significantly, and the overall gray value is higher than the other reconstruction methods. The reconstruction image is not only the smoothness, but also the edge texture processing is also very clear. So the improved algorithm is better than the other five kinds of interpolation algorithm.

\section{Conclusion}

In this paper, a resolution enhancement method based on feature space is proposed. In this method the autocorrelation of the image is assumed. According to experimental results, the resolution enhancement method is superior to the nearest neighbor interpolation and Bilinear interpolation. It is effective in the corresponding region, and it can enhance the visual effect in high frequency detail area.

\section{Acknowledgements}

This work was supported by the Research Foundation of Education Bureau of Jilin Province under the Grant Number: 2015254.

\section{References}

[1] F. Šroubek and J. Flusser: Resolution enhancement via probabilistic deconvolution of multiple degraded images, Pattern Recognition Letters, Vol.27 (2006) No.4, p.287 - 293.

[2] C. Bi,L. Geng and X. Zhang: Cubic spline interpolation-based time-domain equivalent source method for modeling transient acoustic radiation, Journal of Sound and Vibration, Vol.332 (2013) No.22, p.5939 - 5952.

[3] M. A. Navascués and M. V. Sebastián: Generalization of Hermite functions by fractal interpolation, Journal of Approximation Theory, Vol.131 (2004) No.1, p.19 - 29.

[4] V. D. Nguyen,E. Béchet and C. Geuzaine: Imposing periodic boundary condition on arbitrary meshes by polynomial interpolation, Computational Materials Science, Vol.55 (2012) No."", p.390 - 406 . 
[5] S. Hu and P. Shao: Improved nearest neighbor interpolators based on confidence region in medical image registration, Biomedical Signal Processing and Control, Vol.7 (2012) No.5, p.525 - 536.

[6] M. Abbas,A. A. Majid and J. M. Ali: Positivity-preserving rational bi-cubic spline interpolation for 3D positive data, Applied Mathematics and Computation, Vol.234 (2014) No."", p.460 - 476.

[7] H. Chen and J. Leou: Saliency-directed image interpolation using particle swarm optimization, Signal Processing, Vol.90 (2010) No.5, p.1676 - 1692.

[8] X. Liu,G. R. Liu and K. Tai: Radial point interpolation collocation method (RPICM) for partial differential equations, Computers \& Mathematics with Applications, Vol.50 (2005) No.8 - 9 , p.1425 - 1442.

[9] E. H. W. Meijering,W. J. Niessen and M. A. Viergever: Quantitative evaluation of convolution-based methods for medical image interpolation, Medical Image Analysis, Vol.5 (2001) No.2, p.111 - 126.

[10] C. Lin,Y. Du Du and T. Chen: Nonlinear interpolation fractal classifier for multiple cardiac arrhythmias recognition, Chaos, Solitons \& Fractals, Vol.42 (2009) No.4, p.2570 - 2581. 